\title{
Isolated Premature Menarche
}

National Cancer Institute

\section{Source}

National Cancer Institute. Isolated Premature Menarche. NCI Thesaurus. Code C120164.

Occurrence of the first menstrual period in a girl before the lower limit of the normal age range for the reference population, without other signs of puberty. 